\title{
Nachhaltiges Projektmanagement am Beispiel der Einführung von Enterprise Resource Planning-Software
}

\author{
Bertil Haack
}

Zusammenfassung

Obwohl »Nachhaltigkeit« beinahe schon ein Modewort ist, gibt es bisher nur sehr wenige Anstrengungen in Richtung auf ein nachhaltiges Projektmanagement. Der vorliegende Beitrag dient dazu, einen praktikablen Vorschlag für nachhaltiges Projektmanagement am Beispiel der Einführung von Enterprise Resource PlanningSoftware (ERP) zu entwickeln. Darin wird das klassische Projektmanagement in zwei Schritten zum nachhaltigen Projektmanagement ausgeformt. Das Ergebnis kann auf andere als die betrachteten Projekte übertragen werden.
Abstract

"Sustainability« is nearly a buzzword. Nevertheless activities concerning sustainable project management are few in number. With regard to the implementation of Enterprise Resource Planning Software (ERP) this paper offers a feasible proposal for sustainable project management. Starting with classical project management two steps are necessary to get the sustainable project management concept which can also be transferred to other types of projects.

\section{Einleitung}

Die Forderung, nachhaltig zu agieren, wird mittlerweile in nahezu allen beruflichen und sehr vielen privaten Bereichen aufgestellt. Abgesehen davon, dass Nachhaltigkeit damit bedauerlicherweise auch den Charakter eines Modeworts und Trends gewonnen hat, stellt sich die Frage, wie Nachhaltigkeit in der Praxis realisiert werden kann. Diese ist im Bereich des Projektmanagements bisher eher nur rudimentär beantwortet worden (vgl. etwa Sustainable-Project-Management 2011).

Der vorliegende Beitrag dient dazu, einen praktikablen Ansatz für nachhaltiges Projektmanagement am Beispiel der Einführung von Enterprise Resource Planning-Software (ERP) zu entwickeln.

Dazu wird zunächst die Ausgangsposition der Überlegungen geklärt (Kapitel 2). Anschließend wird das klassische Projektmanagement skizziert und in zwei Schritten zum nachhaltigen Projektmanagement ausgeformt (Kapitel 3 bis 5). Die Ausführungen enden mit einer Diskussion des vorgeschlagenen Projektmanagementkonzeptes (Kapitel 6).

\section{Ausgangsposition}

Projekte sind nahezu allgegenwärtig in der beruflichen Praxis, sodass systematisches Projektmanagement mehr und mehr als kritischer Erfolgsfaktor betrachtet werden muss, der wesentlich zum erfolgreichen Agieren und Überleben von Unternehmen am Markt beiträgt (vgl. etwa Köster 2010: xxii; Kerzner 2006: xix).

Dies gilt auch und gerade für den Bereich der in Unternehmen erforderlichen IT-Systeme und dort insbesondere für die Einführung von SAP als dem Vertreter von ERP-Systemen weltweit (Gartner Group 2011): »Alle etablierten SAP-Beratungsunternehmen beherrschen gleichermaßen die Einführung der ERP-Software SAP bei ihren Kunden. Sehr gutes Projektmanagement ist daher ein wichtiges Differenzierungskriterium eines SAP-Beratungsunternehmens, um sich langfristig am Markt behaupten zu können. (Schulz 2011) Entsprechend wird hier das Augenmerk auf das Projektmanagement bei der Einführung von SAP gerichtet. 
Projekt (DIN 69901: Durch Einmaligkeit der Bedingungen gekennzeichnetes Vorhaben)

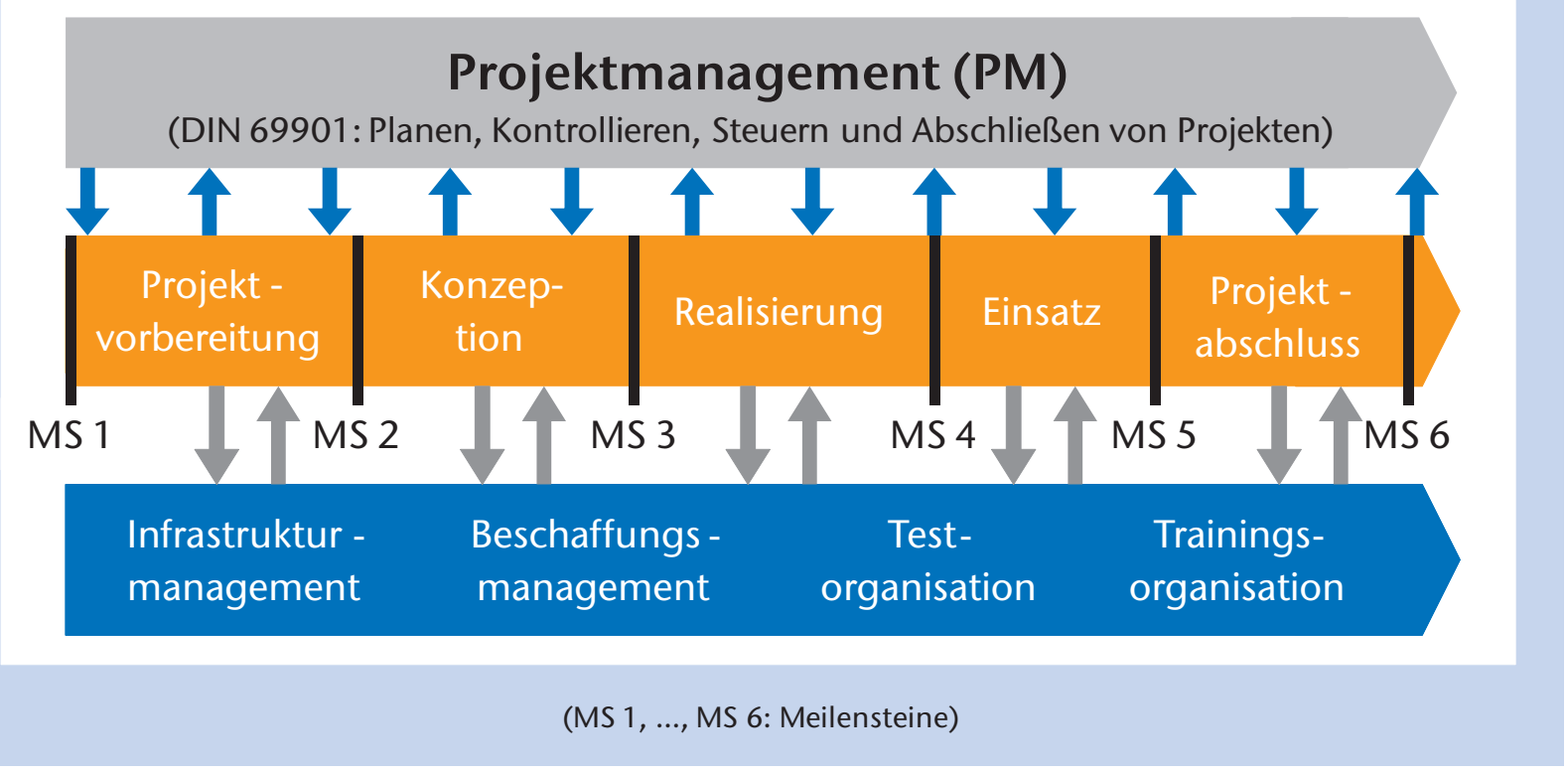

Abb. 1: Allgemeines Phasenmodell für Projekte (eigene Darstellung in Anlehnung an Gadatsch 2008: 49)

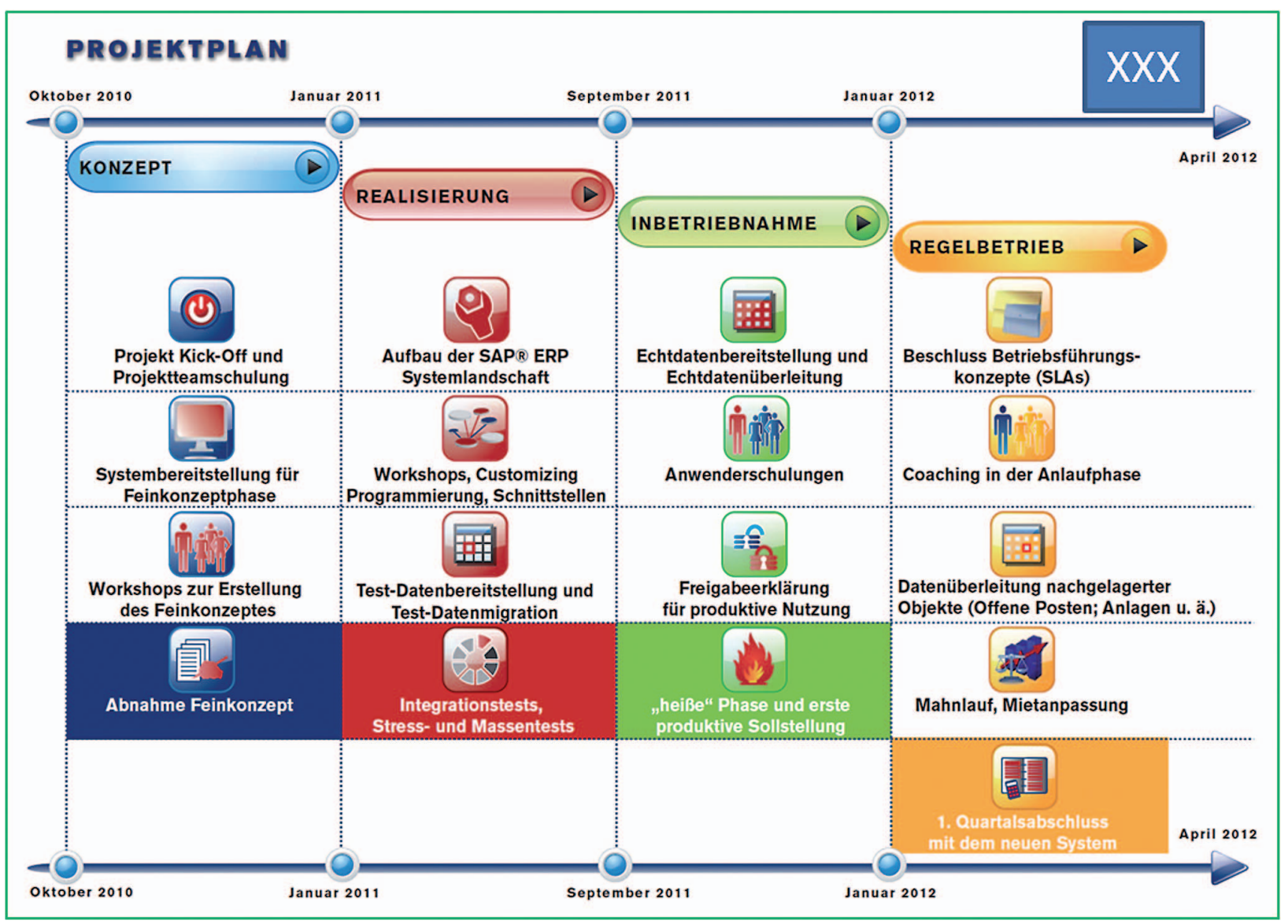

Abb. 2: Projektplan zur Einführung von SAP im Liegenschaftsbetrieb XXX (PROMOS consult 2011) 


\section{Klassisches Projektmanagement bei der ERP-Einführung}

Die Implementierung von ERP-Software in einem Unternehmen ist ein durch die Einmaligkeit seiner Bedingungen gekennzeichnetes Vorhaben, erfüllt also die Kriterien für ein Projekt gemäß DIN 69901 (Beuth 2011) und kann durch ein Vorgehen entsprechend Abbildung 1 realisiert werden.

In der Praxis stellt sich dieses Vorgehen etwa wie in Abbildung 2 dar. Hierbei handelt es sich um den Projektplan per Februar 2011 der PROMOS consult zur Einführung von SAP bei einer größeren Liegenschaftsverwaltung XXX (Name auf Wunsch anonymisiert) in der Bundesrepublik Deutschland. Die PROMOS consult selbst, Kooperationspartner der TH Wildau, gehört zu den Marktführern in Deutschland im Bereich der Einführung und des Betriebs von SAP-Systemen.

Dieser Projektplan verweist auf die klassischen Projektmanagementziele:

- Ressourcen/ Kosten

- Zeit

- Leistung

Eine bestimmte Leistung soll im Rahmen eines festgelegten Budgets in einer vorgegebenen Zeit erbracht werden (s. Abbildung 3).

\section{Erweitertes Projektmanagement bei der ERP-Einführung}

SAP selbst schlägt mit dem sogenannten Accelerated SAP (ASAP) ein etwas komplexeres Phasenmodell zur SAP-Einführung vor. Dieses unterscheidet sich vom klassischen Phasenmodell (Abbildung 1) bzw. vom Ansatz der PROMOS consult (Abbildungen 2 und 3) vor allem dadurch, dass es drei weitere Dimensionen aufzeigt, mit denen Projektmanagemententscheidungen im Wechsel wirken:

- Program Management

- Risk Management

- Quality Management

Projektmanager müssen danach auch die Beziehungen ihres Projekts zu anderen Vorhaben im Unternehmen wie beispielsweise Organisationsprojekte zur Veränderung von Geschäftsprozessen, die möglichen Projektrisiken und das Qualitätsmanagement bewusst in ihre Überlegungen und Entscheidungen einbeziehen (s. Abbildung 4).

Ein in diesem Sinne erweitertes Projektmanagement muss also mindestens sechs Zieldimensionen in Einklang bringen. Gleichwohl weist es in Bezug auf die eingangs gestellte Frage nach wie vor ein wesentliches Manko auf: ein Projektmanagement gemäß dem klas-

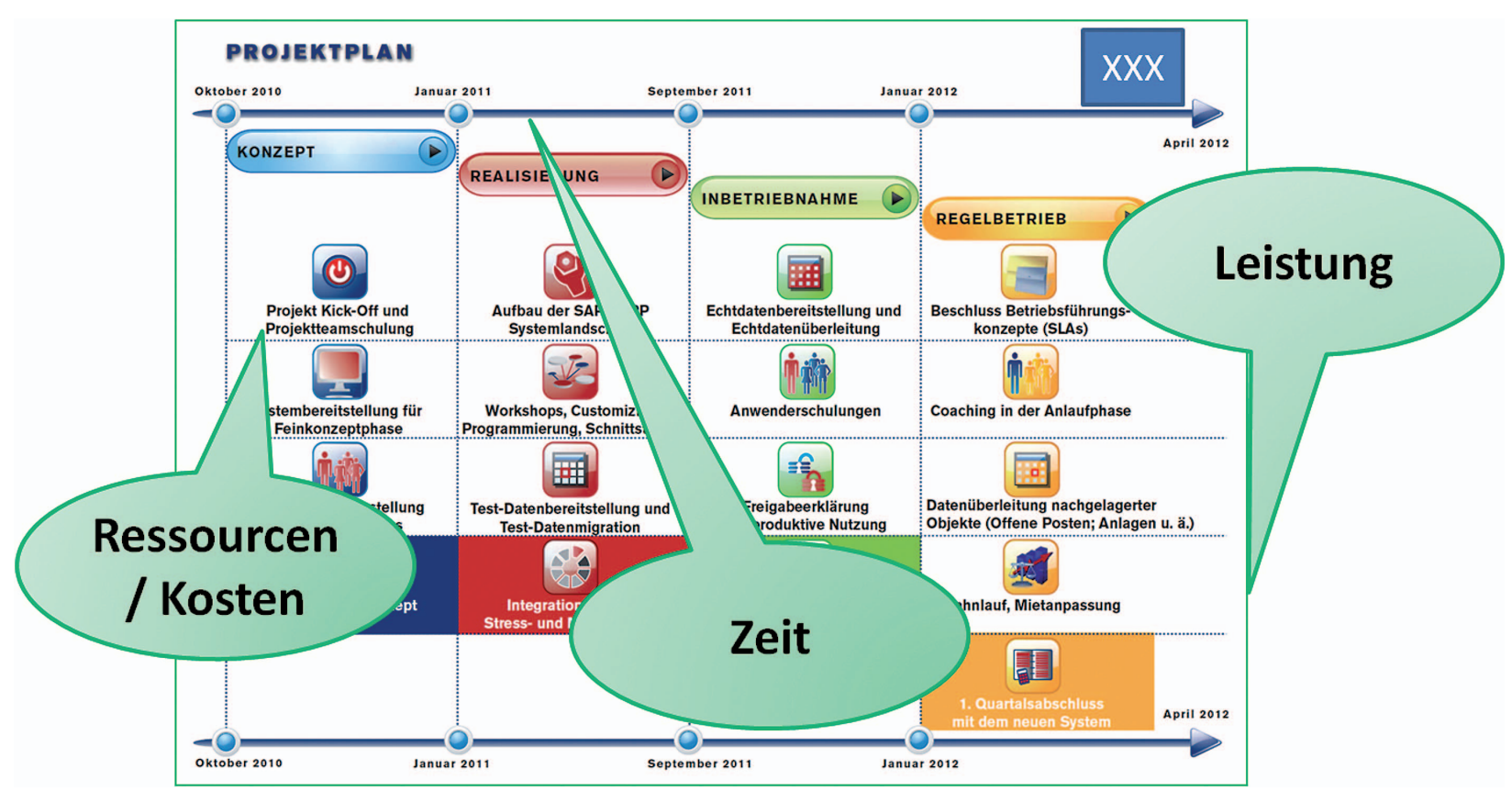

Abb. 3: Klassische Projektziele bei der SAP-Einführung im Liegenschaftsbetrieb XXX (eigene Darstellung in Anlehnung an PROMOS consult 2011) 


\begin{tabular}{l}
\hline Program Management \\
\hline Project Management \\
\hline Risk Management \\
\hline Quality Management \\
\hline Business Process Requirements, Design, Configuration and Testing \\
\hline Content Management and User Interface \\
\hline Developments \\
\hline Security, Job Roles and System Authorizations \\
\hline Lifecycle Data Management \\
\hline Technical Solution Management \\
\hline SAP Safeguarding \\
\hline Organizational Change Management and Training
\end{tabular}

Abb. 4: Projektmodell Accelerated SAP (ASAP) der SAP AG (eigene Darstellung in Anlehnung an Stöckmann 2011 und Becker et al. $2008: 336 \mathrm{ff}$.

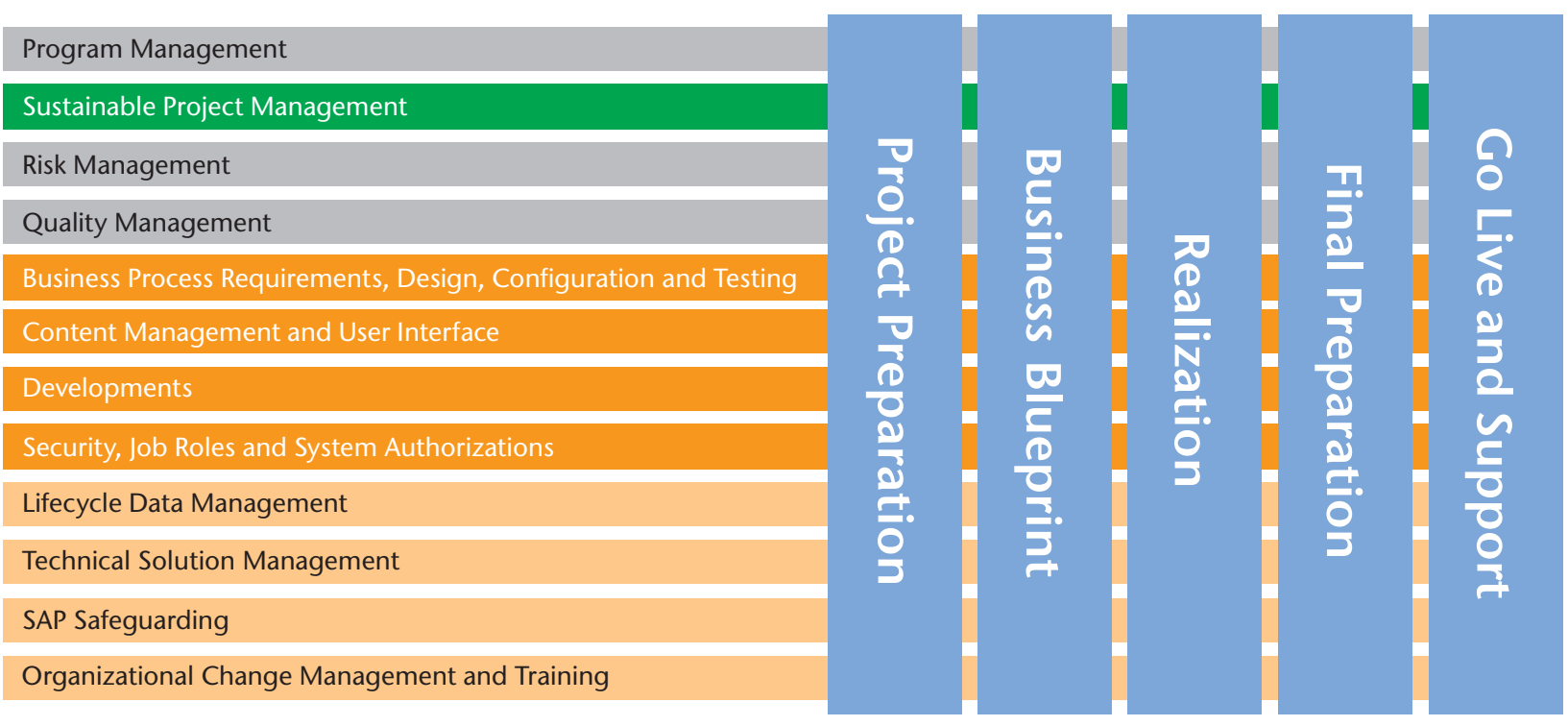

Abb. 5: Projektmodell Accelerated Sustainable SAP (AS²AP) (eigene Darstellung in Anlehnung an Stöckmann 2011 und Becker et al. 2008: $336 \mathrm{ff}$ )

sischen und dem erweiterten Projektmodell nimmt Umweltauswirkungen des Handelns kaum in den Blick - ein Sachverhalt, der nicht zuletzt durch die Erfahrungen des Autors aus über 20 Jahren Projektarbeit im ITUmfeld bestätigt wird!

Gerade die klassischen Zieldimensionen führen dazu, dass der Fokus des Projektmanagements auf das Projektende ausgerichtet ist. Es kommt darauf an, die Projektvorgaben einzuhalten und die gewünschte Leistung zur richtigen Zeit zu erbringen, ohne den finanziellen Rahmen für das Projekt zu sprengen.

Fragen wie etwa die nach der Vermeidung von Emissionen aufgrund von Dienstreisen oder die nach der Reduktion von Ressourcenverbräuchen werden zumin- dest im IT-Bereich in der Regel ebenso selten gestellt wie die nach den Auswirkungen des Projektergebnisses auf die Umwelt.

Dabei zeigt beispielsweise die Diskussion der Klimaschädlichkeit von Google-Anfragen (eCommerce 2011), dass jede Nutzung von IT-Systemen Auswirkungen auf die Umwelt hat. Diese sind durch das Design der Projektlösung so weit wie möglich zu reduzieren. Das kann nur in der Projektlaufzeit geschehen, da die Projektlösung in eben jener Zeit entwickelt wird und damit Konsequenzen für deren Nutzung im Anschluss an das Projekt festgelegt werden. Entsprechend ist eine Ausweitung der Zieldimensionen des Projektmanagements auf den Komplex »Nachhaltigkeit« erforderlich. 


\section{Nachhaltiges Projektmanagement bei der ERP-Einführung}

Um diese Erweiterung leisten zu können, ist zunächst das Verständnis von »Nachhaltigkeit« (»sustainability«) zu klären. Hier hat sich die Sicht der BrundtlandKommission durchgesetzt: »Im übergreifenden Sinne bewertet >Nachhaltigkeit gegenwärtiges Denken und Handeln unter dem Gesichtspunkt, die Lebenssituation der heutigen Generationen zu verbessern, ohne die Zukunftsperspektiven der kommenden Generationen $\mathrm{zu}$ verschlechtern. [...] Ziel [...] ist die Sicherstellung und Verbesserung ökologischer, ökonomischer und sozialer Leistungsfähigkeit. Diese bedingen einander [...] und können nicht >teiloptimiert « werden. « (Deutscher Bundestag 2004)

Damit bedeutet die Einbeziehung von Nachhaltigkeit in das Projektmanagement, die Zielstellungen

- ökonomische Nachhaltigkeit,

- ökologische Nachhaltigkeit und

- soziale Nachhaltigkeit

simultan mit den vorab genannten sechs Projektmanagementdimensionen $\mathrm{zu}$ berücksichtigen und mit jenen zu harmonisieren.

Entsprechend wird vorgeschlagen, das Projektmodell ASAP zur SAP-Einführung, wie in Abbildung 5 dargestellt, um den Aspekt sustainability zum Projektmodell Accelerated Sustainable SAP (AS $\left.{ }^{2} \mathrm{AP}\right)$ zu ergänzen und so den gesuchten Ansatz für ein nachhaltiges Projektmanagement am Beispiel der Einführung von Enterprise Resource Planning-Software (ERP) zu liefern.

Konkrete Maßnahmen derart nachhaltigen Projektmanagements können beispielsweise darin bestehen, so weit möglich

- Green IT als Hardware-Basis für den Betrieb der ERPLösung auszuwählen,

- Projektteams aus lokal in der Nähe des Projektorts ansässigen Mitarbeitern zu bilden,

n Dienstreisen durch Telefon- oder Videokonferenzen zu ersetzen sowie

- Ausdrucke von Unterlagen zu vermeiden oder mindestens zwei Seiten auf ein Blatt zu drucken.

\section{Diskussion}

Offenbar bietet Accelerated Sustainable SAP (AS²AP) konkrete Maßnahmen, wie Nachhaltigkeit in das Projektgeschehen einbezogen werden kann. Gleichwohl stellt sich die Frage, ob es für diese Ideen wie für den Einsatz von Green IT und die Nutzung von Videokonferenzen eines Modells wie $\mathrm{AS}^{2} \mathrm{AP}$ bedarf. Diese Frage kann auch so formuliert werden, ob die beschriebene Einbeziehung von Nachhaltigkeit in das Projektmanagement eine triviale oder eine nicht-triviale Ausweitung des ursprünglichen Ansatzes ASAP und damit auch des klassischen Projektmanagements darstellt. Hierauf ist zum aktuellen Zeitpunkt keine abschließende Antwort möglich. Dazu sind weitere Forschungsaktivitäten erforderlich. Die Anstrengungen seitens der Sustainable Project Management-Initiatoren, den Projektmanagement-Standard PMBoK um Nachhaltigkeitsaspekte zu ergänzen (Sustainable-Project-Management 2011), geben Anlass für vorsichtigen Optimismus.

Unabhängig davon hat $\mathrm{AS}^{2} \mathrm{AP}$ eine gewisse Komplexität und einen Umfang, die die Anwendung des Projektmodells zumal in weniger problematischen Projektumfeldern wie vielleicht bei der SAP-Einführung in einem kleineren Unternehmen in Frage stellen. Die Erfahrung zeigt, dass auch ausgereifte Projektansätze in der Praxis häufig als zu schwer zu handhaben, zu zeitintensiv o. ä. eingeschätzt und entsprechend zurückhaltend eingesetzt werden. Hier gilt, dass das Projektmodell in jedem Einzelfall sinnvoll taylorisiert, d. h. auf die Projektbelange zugeschnitten, werden kann und soll.

Darüber hinaus ist es notwendig, sich Gedanken zur Werkzeugunterstützung für $\mathrm{AS}^{2} \mathrm{AP} \mathrm{zu}$ machen. Es ist also noch zu klären, welche Tools erforderlich und geeignet sind, die Ausrichtung von Projekten auf ökonomische, ökologische und soziale Nachhaltigkeitsziele zu unterstützen.

Schließlich stellt sich die Frage, ob die vorliegenden Gedanken auch auf andere Projekte in anderen Bereichen übertragen und Nachhaltigkeit damit zum Gegenstand jedes Projektmodells werden kann. Das kann bejaht werden, da die Ausführungen keine Einschränkungen diesbezüglich aufweisen. 


\section{Literatur}

Becker, J., Kugeler, M., Rosemann, M. (Hg.) (2008): Prozessmanagement: Ein Leitfaden zur prozessorientierten Organisationsgestaltung. Springer Verlag, Berlin.

Beuth (2011): Projektmanagement - Projektmanagementsysteme - Teil 1: Grundlagen. http://www.beuth.de/langanzeige/DIN69901-1/de/113428320.html, Zugriff: 10.08.2011.

Deutscher Bundestag (2004): Wissenschaftliche Dienste des Deutschen Bundestages: Nachhaltigkeit. Der aktuelle Begriff 06/2004, 6. April 2004, Berlin.

eCommerce (2011): Google als Klimasünder beschuldigt. http:// www.e-commerce-magazin.de/ecm/news/google-als-klimasuender-beschuldigt, Zugriff: 10.08.2011.

Gadatsch, A. (2008): Grundkurs Geschäftsprozess-Management: Methoden und Werkzeuge für die IT-Praxis. Eine Einführung für Studenten und Praktiker, Vieweg Verlag, Wiesbaden.

Gartner Group (2011): Gartner Says Worldwide Business Intelligence, Analytics and Performance Management Software Market Grew 4 Percent in 2009, http://www.gartner.com/it/page. jsp?id=1357514, Zugriff: 10.08.2011.

Kerzner, H. (2006): Project Management - A Systems Approach to Planning, Scheduling, and Controlling. John Wiley \& Sons, Inc., Hoboken (NJ).

Köster, K. (2010): International Project Management. SAGE Publications Ltd, London.

PROMOS consult (2011): Projektplan der PROMOS consult GmbH zur Einführung von SAP im Unternehmen XXX. PROMOS consult, Berlin.

Schulz, V. (2011): Interview. Geschäftsführer der PROMOS consult $\mathrm{GmbH}$, Berlin.

Stöckmann (2011): Vorgehensmodelle zur Einführung von ERP Software: AcceleratedSAP (ASAP) und Accenture Delivery Methods (ADM). http://www.iwi.uni-hannover.de/lv/seminar_ws06_07_ de/Stoeckmann/files/pdf.pdf, Zugriff: 10.08.2011.

Sustainable-Project-Management (2011): Sustainable Project Management. http://www.sustainable-project-management.org/cms/, Zugriff: 10.08.2011.

\section{Autor}

Prof. Dr. Bertil Haack

Fachbereich Wirtschaft, Verwaltung und Recht

Technische Hochschule Wildau [FH]

$\mathrm{T}+493375$ 508-914

bertil.haack@th-wildau.de 